\title{
Boundedness of Global Solutions for Nonlinear Parabolic Equations Involving Gradient Blow-up Phenomena
}

\author{
JOSÉ M. ARRIETA - ANIBAL RODRIGUEZ-BERNAL - \\ PHILIPPE SOUPLET
}

\begin{abstract}
We consider a one-dimensional semilinear parabolic equation with a gradient nonlinearity. We provide a complete classification of large time behavior of the classical solutions $u$ : either the space derivative $u_{x}$ blows up in finite time (with $u$ itself remaining bounded), or $u$ is global and converges in $C^{1}$ norm to the unique steady state.

The main difficulty is to prove $C^{1}$ boundedness of all global solutions. To do so, we explicitly compute a nontrivial Lyapunov functional by carrying out the method of Zelenyak. After deriving precise estimates on the solutions and on the Lyapunov functional, we proceed by contradiction by showing that any $C^{1}$ unbounded global solution should converge to a singular stationary solution, which does not exist. As a consequence of our results, we exhibit the following interesting situation: - the trajectories starting from some bounded set of initial data in $C^{1}$ describe an unbounded set, although each of them is individually bounded and converges to the same limit;

- the existence time $T^{*}$ is not a continuous function of the initial data.
\end{abstract}

Mathematics Subject Classification (2000): 35K60, 35K65, 35B45.

\section{1. - Introduction and main results}

We consider the problem

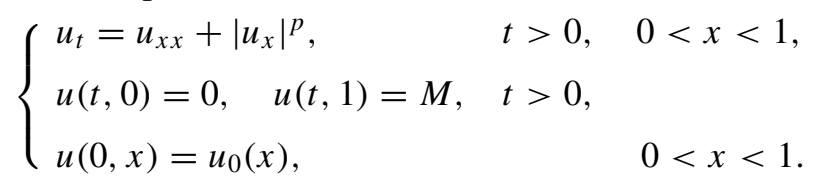

Here $p>2, M \geq 0$ and $u_{0} \in X$, where $X=\left\{v \in C^{1}([0,1]) ; v(0)=0, v(1)=\right.$ $M$ \}, endowed with the $C^{1}$ norm. The problem (1.1) admits a unique maximal

Partially supported by Project BFM2000-0798, DGES Spain.

Pervenuto alla Redazione il 3 luglio 2003. 
classical solution $u=u\left(u_{0} ; t,.\right)$, whose existence time will be denoted by $T^{*}=T^{*}\left(u_{0}\right) \in(0, \infty]$. Note that we make no restriction on the signs of $u$ or $u_{x}$.

The equation (1.1) possesses both mathematical and physical interest. It can serve as a typical model-case in the theory of parabolic PDEs. Indeed, it is the simplest example of a parabolic equation with a nonlinearity depending on the first order spatial derivatives of $u$, and it can be considered as an analogue of the extensively studied equation with zero order nonlinearity $u_{t}-u_{x x}=u^{p}$. On the other hand, the equation (1.1) (and its $N$ dimensional version) arises in the viscosity approximation of Hamilton-Jacobi type equations from stochastic control theory [21] and in some physical models of surface growth [18]. A rather up-to-date list of references can be found in [6] (especially for the Cauchy problem, which has been more studied). For studies concerning the Dirichlet and Neumann problems, we also refer to [2], [9], [1], [28], [4], [5], [10].

The aim of this paper is to provide a complete classification of large time behavior of the solutions of (1.1). A basic fact about (1.1) is that the solutions satisfy a maximum principle:

$$
\min _{[0,1]} u_{0} \leq u(t, x) \leq \max _{[0,1]} u_{0}, \quad 0 \leq t<T^{*}, \quad 0 \leq x \leq 1
$$

Since (1.1) is well-posed in $C^{1}$, therefore only three possibilities can occur:

(I) $u$ exists globally and is bounded in $C^{1}$ :

$$
T^{*}=\infty \quad \text { and } \quad \sup _{t \geq 0}\left|u_{x}(t, .)\right|_{\infty}<\infty
$$

Moreover, due to the results in [30] (see the last part of this Introduction for more details), $u$ has to converge in $C^{1}$ to a steady state (which is actually unique when it exists);

(II) $u$ blows up in finite time in $C^{1}$ norm (finite time gradient blow-up):

$$
T^{*}<\infty \quad \text { and } \quad \lim _{t \rightarrow T^{*}}\left|u_{x}(t, .)\right|_{\infty}=\infty
$$

(III) $u$ exists globally but is unbounded in $C^{1}$ (infinite time gradient blow-up):

$$
T^{*}=\infty \quad \text { and } \quad \limsup _{t \rightarrow \infty}\left|u_{x}(t, .)\right|_{\infty}=\infty
$$

For simplicity, let us first consider the case $M=0$. When $\left|u_{0}\right|_{C^{1}}$ is sufficiently small, then it is known that (I) occurs (and $u$ converges to the unique steady state $\left.V_{0} \equiv 0\right)$. If, on the contrary, $u_{0}$ is suitably large, then (II) occurs (see [28]).

Our primary goal is therefore to rule out (III), that is, infinite time gradient blow-up. 
For $M>0$, the situation is slightly more involved. There exists a critical value

$$
M_{c}=\frac{(p-1)^{\frac{p-2}{p-1}}}{p-2}
$$

such that (1.1) has a unique (regular) steady state $V_{M}$ if $M<M_{c}$ and no steady state if $M>M_{c}$ (the explicit formula for $V_{M}$ is recalled at the beginning of Section 2). In the critical case $M=M_{c}$, there still exists a steady state $V_{M_{c}}$, but it is singular, satisfying $V_{M_{c}} \in C([0,1]) \cap C^{2}((0,1])$ with $V_{M_{c}, x}(0)=\infty$. Restricting to initial data $u_{0}$ such that $u_{0}$ and $u_{0, x} \geq 0$, it was shown among other things in [2] that:

(i) if $M>M_{c}$, then all solutions of (1.1) satisfy (II);

(ii) if $0<M<M_{c}$, then both (I) and (II) occur. However the possibility of (III) remained as an open problem.

Therefore, our main interest lies in the case $M<M_{c}$. Our main result is the following.

THeOREM 1. Assume $0 \leq M<M_{c}$. Then all global solutions of (1.1) are bounded in $C^{1}$. In other words, (III) cannot occur. Moreover, they converge in $C^{1}$ norm to $V_{M}$.

For the case $M>M_{c}$, we improve the result of [2] by removing the restrictions $u_{0} \geq 0$ and $u_{0, x} \geq 0$ on the initial data.

Proposition 2. Assume $M>M_{c}$. Then all solutions of (1.1) blow up in finite time in $C^{1}$ norm.

Remarks 1. (a) In the critical case $M=M_{c}$, all solutions have to blow up in $C^{1}$ in either finite or infinite time. Moreover, if (III) occurs, then the solution will converge in $C([0,1])$ to the singular steady state $V_{M_{c}}$, as $t \rightarrow \infty$. This follows from Proposition 3.2 below. However, the possibility of (III) remains an open problem in this case. We conjecture that this could occur ${ }^{(1)}$.

(b) For $0<p \leq 2$, the situation is quite different. It is known from the local theory (cf. $[19,20]$ ) that a bound on $u$ implies a bound on $u_{x}$. Therefore all solutions are global and bounded in $C^{1}$, and they converge to the unique steady state.

(c) For results on the asymptotic behavior of solutions of (1.1) with finite time gradient blow-up, we refer to [9] (see also [3], [13] for variants of (1.1)).

As a consequence of our results, we exhibit the following interesting situation: although $C^{1}$ boundedness of global solutions is true, the global solutions of (1.1) do not satisfy a uniform a priori estimate, i.e., the supremum in (I) cannot be estimated in terms of the norm of the initial data. In other words, there exists a bounded, even compact, subset $\mathcal{S} \subset X$, such that the trajectories

(1) After the completion of this paper, a positive answer to this conjecture was given to us by Juan-Luis Vázquez (personal communication). 
starting from $\mathcal{S}$ describe an unbounded subset of $X$, although each of them is individually bounded and converges to the same limit. As a further consequence, the existence time $T^{*}$, defined as a function from $X$ into $(0, \infty]$, is not (upper semi-)continuous.

Proposition 3. Assume $0 \leq M<M_{c}$. There exists $u_{0} \in X$ and a sequence $\left(u_{0, n}\right)$ in $X$ with the following properties.

(i) $u_{0, n} \rightarrow u_{0}$ in $C^{1}$;

(ii) $T^{*}\left(u_{0, n}\right)=\infty$ for each $n$, and $T^{*}\left(u_{0}\right)<\infty$;

(iii) $\sup _{t \geq 0}\left|u_{n, x}(t, .)\right|_{\infty}=: K_{n} \rightarrow \infty$.

REMARKS 2. (a) The questions of boundedness and a priori estimates of global solutions have been intensively studied for the zero order reactiondiffusion equation

$$
u_{t}=u_{x x}+|u|^{p-1} u
$$

with $p>1$ and homogeneous Dirichlet boundary conditions. The situation is different from ours: all global solutions are not only bounded in $L^{\infty}$ [7], but they also satisfy a uniform a priori estimate depending only on $\left|u_{0}\right|_{\infty}$ [17], [23]. Moreover, the function $T^{*}: X=C_{0}(0,1) \rightarrow(0, \infty]$ is continuous (see [25] and the references therein). Recently, for global positive solutions, it was even shown [15], [24], [26] that at any given $t>0$, the bound is actually universal, i.e. independent of the solution. These results remain true for the $n$ dimensional version of $(1.3)$ for subcritical $p\left(p<p_{S}:=(n+2) /(n-2)_{+}\right)$, but they may fail if $p \geq p_{S}$ [22], [16]. In particular in the radial case [16], for $p$ in a certain interval $\left(p_{S}, p^{*}\right)$, the situation is similar to (1.1) (global solutions are bounded but they fail to satisfy an a priori estimate and $T^{*}$ is discontinuous), whereas for $p=p_{S}$, some global unbounded solutions coexist with global bounded and finite time blow-up solutions.

(b) Set $\mathcal{G}=\left\{u_{0} \in X ; T^{*}\left(u_{0}\right)=\infty\right\}$ and $\mathcal{B}=\left\{u_{0} \in X ; T^{*}\left(u_{0}\right)<\infty\right\}$. Then, we have $\mathcal{G} \cup \mathcal{B}=X$ for both (1.1) and (1.3) (with e.g. $X=C_{0}(0,1)$ in the latter case). However, $\mathcal{G}$ is closed for (1.3) (cf. [7]), while $\mathcal{G}$ is open for (1.1) (this follows easily from the proof of Proposition 3).

REMARKS 3. (a) Let us mention some results related to ours, regarding other parabolic equations with gradient terms. The semilinear equation $u_{t}-\Delta u=$ $u^{p}-\lambda|\nabla u|^{q}(p, q>1, \lambda>0)$ has been studied by several authors (see [27] for a recent survey). In particular, it was proved in [11], [29] that for certain $p$ (even in one space dimension), this equation possesses some global solutions which become unbounded in $L^{\infty}$, as $t \rightarrow \infty$. In some cases, it is even known [11] that these solutions coexist with global bounded and finite time blow-up solutions. For quasilinear equations involving mean curvature type operators, like $u_{t}=\left(\left(1+u_{x}^{2}\right)^{-1 / 2} u_{x}\right)_{x}+\lambda u(\lambda>0)$, a phenomenon of infinite time gradient blow-up (with $u$ remaining bounded) has been exhibited in [8], thus showing a situation opposite to ours. For the convective-reactive problem 
$u_{t}-u_{x x}=u^{p}-\lambda\left(u^{m}\right)_{x}$, the question of boundedness of global solutions is still open, but partial results in this direction may be found in [14].

(b) For singular problems of the form $u_{t}-u_{x x}=\lambda(1-u)^{-k}(k>1, \lambda>0)$, it has been proved in [12], for increasing solutions, that quenching, i.e. blow-up of $u_{t}$ (when $u$ reaches 1 ), can occur only in finite time.

To explain the ideas of our proof, let us first recall that in a classical paper [30], Zelenyak showed that any one-dimensional quasilinear uniformly parabolic equation possesses a (strict) Lyapunov functional, of the form

$$
\mathcal{L}(u(t))=\int_{0}^{1} \phi\left(u(t, x), u_{x}(t, x)\right) d x .
$$

The construction of $\phi$ is in principle explicit, although too complicated to be completely computed in most situations. As a consequence, for any solution $u$ of (1.1) which is global and bounded in $C^{1}$, the (non-empty) $\omega$-limit set of $u$ consists of equilibria. Since (1.1) admits at most one equilibrium $V$, such $u$ has to converge to $V$. (In fact, it was also proved in [30] that whether or not equilibria are unique, any bounded solution of a one-dimensional uniformly parabolic equation converges to an equilibrium; but this need not concern us here.) For $M>0$, our proof proceeds by contradiction and makes essential use of the Zelenyak construction (for the case $M=0$, the proof is actually simpler and does not require the Liapunov functional - see the end of Section 2). It consists of three steps:

- Assuming that a $C^{1}$ unbounded global solution would exist, we analyze its possible final singularities (along a sequence $t_{n} \rightarrow \infty$ ). We shall show that $u_{x}$ remains bounded away from the left boundary and describe the shape of $u_{x}$ near the boundary (cf. Section 2).

- We shall carry out the Zelenyak construction in a sufficiently precise way to determine the density $\phi(u, v)$ of the Lyapunov functional. It will turn out that, whenever $u$ remains in a bounded set of $\mathbb{R}$ (as it does here in view of the estimate (1.2)), $\phi(u, v)$ remains bounded from below uniformly w.r.t. $v$ (see Proposition 3.1).

- Using this property of $\phi$ in the classical Lyapunov argument, together with the fact that singularities may occur only near the boundary, it will be possible to prove the following convergence result: any global solution, even unbounded in $C^{1}$, has to converge in $C([0,1])$ to a stationary solution $W$ of (1.1) with $W(0)=0, W(1)=M$ (see Proposition 3.2). On the other hand, if $u$ were unbounded, then our estimates would imply $W_{x}(0)=\infty$. But such a $W$ is not available if $M \neq M_{c}$, leading to a contradiction.

AcKnowledgement. Part of this research was carried out when the third author was visiting the Universidad Complutense de Madrid. He thanks this institution for its hospitality. 


\section{2. - Preliminary estimates and proof of Theorem 1 for $M=0$}

Let us recall that the only solutions $v \in C([0,1]) \cap C^{2}(0,1)$ of

$$
\left\{\begin{array}{l}
v_{x x}+\left|v_{x}\right|^{p}=0, \quad 0<x<1 \\
v(0)=0
\end{array}\right.
$$

are given by $v=0$ or $v=v_{k}(x):=M_{c}\left((x+k)^{\frac{p-2}{p-1}}-k^{\frac{p-2}{p-1}}\right), k \in[0, \infty)$. Moreover, $v_{k}(1)$ (resp., $v_{k, x}(0)$ ) increases from 0 to $M_{c}$ (resp., 0 to $\infty$ ), as $k$ decreases from $\infty$ to 0 . In particular, for each $M \in\left(0, M_{c}\right), V_{M}=v_{k(M)}$ for a unique $k(M) \in(0, \infty)$.

We start with some preliminary estimates. They are collected in Lemmas 2.1-2.6.

Lemma 2.1. Let $u$ be a maximal solution of (1.1). For all $t_{0} \in\left(0, T^{*}\right)$, there exists $C_{1}>0$ such that

$$
\left|u_{t}\right| \leq C_{1}, \quad t_{0} \leq t<T^{*}, \quad 0 \leq x \leq 1 .
$$

Proof. The function $h=u_{t}$ satisfies

$$
\begin{cases}h_{t}=h_{x x}+a(t, x) h_{x}, & t_{0}<t<T^{*}, \quad 0<x<1, \\ h(t, 0)=h(t, 1)=0, & t_{0}<t<T^{*}, \\ h\left(t_{0}, x\right)=u_{x x}\left(t_{0}, x\right)+\left|u_{x}\left(t_{0}, x\right)\right|^{p}, & 0<x<1,\end{cases}
$$

where $a(t, x)=p\left|u_{x}\right|^{p-2} u_{x}$. It follows from the maximum principle that $|h| \leq$ $\left|h\left(t_{0}\right)\right|_{\infty}$ in $\left[t_{0}, T^{*}\right) \times[0,1]$.

The following two lemmas give upper and lower bounds on $u_{x}$ which show, in particular, that $u_{x}$ remains bounded away from the boundary.

Lemma 2.2. Let $u$ be a maximal solution of (1.1). For all $t_{0} \in\left(0, T^{*}\right)$, there exists $C_{1}>0$ such that, for all $t_{0} \leq t<T^{*}$ and $0 \leq x \leq 1$,

$$
u_{x}(t, x) \leq C_{1} x+((p-1) x)^{-\frac{1}{p-1}}
$$

and

$$
u_{x}(t, 1-x) \geq-C_{1} x-((p-1) x)^{-\frac{1}{p-1}} .
$$

Proof. Fix $t \in\left[t_{0}, T^{*}\right)$ and let $y(x)=\left(u_{x}(t, x)-C_{1} x\right)_{+}$, where $C_{1}$ is given by Lemma 2.1. The function $y$ satisfies

$$
y^{\prime}+y^{p}=\left(u_{x x}-C_{1}\right) \mathbf{1}_{\left\{u_{x}>C_{1} x\right\}}+\left(u_{x}-C_{1} x\right)_{+}^{p} .
$$

For each $x$ such that $u_{x}(t, x)>C_{1} x$, we have $\left(y^{\prime}+y^{p}\right)(x) \leq\left(u_{x x}-C_{1}+\right.$ $\left.\left|u_{x}\right|^{p}\right)(x) \leq 0$ by Lemma 2.1. Therefore, we have $y^{\prime}+y^{p} \leq 0$ on $(0,1)$. By integration, it follows that $y(x) \leq((p-1) x)^{-\frac{1}{p-1}}$, hence (2.1).

As for (2.2), it follows similarly by considering $y(x)=\left(-u_{x}(t, 1-x)-\right.$ $\left.C_{1} x\right)_{+}$. 
Lemma 2.3. Let $u$ be a maximal solution of (1.1). There exists $C_{2}>0$ such that, for all $T \in\left(0, T^{*}\right)$,

$$
\max _{Q_{T}} u_{x}(t, x) \leq \max \left(C_{2}, \max _{0 \leq t \leq T} u_{x}(t, 0)\right),
$$

where $Q_{T}=[0, T] \times[0,1]$, and

$$
\min _{Q_{T}} u_{x}(t, x) \geq \min \left(-C_{2}, \min _{0 \leq t \leq T} u_{x}(t, 1)\right) .
$$

Proof. The function $w=u_{x}$ satisfies $w_{t}=w_{x x}+a(t, x) w_{x}$ in $\left(0, T^{*}\right) \times$ $(0,1)$, where $a(t, x)=p\left|u_{x}\right|^{p-2} u_{x}$. Therefore, $w$ attains its extrema in $Q_{T}$ on the parabolic boundary of $Q_{T}$.

Since, by Lemma 2.2 , we have $u_{x}(t, 1) \leq C$ and $u_{x}(t, 0) \geq-C$ for all $t \in\left[0, T^{*}\right)$, the conclusion follows.

The following lemma will provide a useful lower bound on the blow-up profile of $u_{x}$ in case $u_{x}(t, 0)$ or $u_{x}(t, 1)$ becomes unbounded.

Lemma 2.4. Let $u$ be a maximal solution of (1.1). For all $t_{0} \in\left(0, T^{*}\right)$, there exists $C_{3}>0$ such that, for all $t_{0} \leq t<T^{*}$ and $0 \leq x \leq 1$,

$$
\left[u_{x}^{+}(t, x)+C_{3}\right]^{1-p} \leq\left[u_{x}^{+}(t, 0)+C_{3}\right]^{1-p}+(p-1) x
$$

and

$$
\left[\left(-u_{x}\right)^{+}(t, 1-x)+C_{3}\right]^{1-p} \leq\left[\left(-u_{x}\right)^{+}(t, 1)+C_{3}\right]^{1-p}+(p-1) x
$$

Proof. Fix $t \in\left[t_{0}, T^{*}\right)$ and let $z(x)=u_{x}^{+}(t, x)+C_{1}^{1 / p}$, where $C_{1}$ is given by Lemma 2.1. The function $z$ satisfies

$$
z^{\prime}+z^{p}=u_{x x} \mathbf{1}_{\left\{u_{x}>0\right\}}+\left(u_{x}^{+}(t, x)+C_{1}^{1 / p}\right)^{p} \geq\left(u_{x x}+\left|u_{x}\right|^{p}\right) \mathbf{1}_{\left\{u_{x}>0\right\}}+C_{1} \geq 0
$$

on $[0,1]$ by Lemma 2.1. By integration, it follows that $z^{1-p}(x) \leq z^{1-p}(0)+$ $(p-1) x$, that is $(2.5)$ with $C_{3}=C_{1}^{1 / p}$.

The estimate (2.6) follows similarly by considering $z(x)=\left(-u_{x}\right)^{+}(t, 1-$ $x)+C_{1}^{1 / p}$.

The following Lemma enables us to rule out infinite time gradient blow-up to $-\infty$ i.e., at $x=1$.

Lemma 2.5. Let u be a global solution of (1.1). Then it holds

$$
\inf _{[0, \infty) \times[0,1]} u_{x}>-\infty
$$

The proof of Lemma 2.5 relies on the following property. 
LEMMA 2.6. Let $u$ be a global solution of (1.1). Then we have

$$
\lim _{t \rightarrow \infty}\left(\max _{x \in[0,1]} u(t, x)\right)=M .
$$

Proof. Fix $w_{0} \in C_{c}^{\infty}(\mathbb{R}), w_{0} \geq 0$, such that $w_{0} \geq u_{0}-M$ on $[0,1]$ and let $w$ be the classical solution of

$$
\begin{cases}w_{t}=w_{x x}+\left|w_{x}\right|^{p}, & 0<t<T, \quad-\infty<x<\infty \\ w(0, x)=w_{0}(x), & -\infty<x<\infty\end{cases}
$$

with $T$ its maximal existence time. By the maximum principle, we have

$$
0 \leq w \leq\left|w_{0}\right|_{\infty} \quad \text { on }(0, T) \times \mathbb{R} \text {. }
$$

Also, since $u-M$ satisfies the differential equation in (1.1) and since $w \geq 0 \geq$ $u-M$ at $x=0,1$ for all $t \in(0, T)$, the comparison principle implies that

$$
w \geq u-M \quad \text { on }(0, T) \times \mathbb{R} \text {. }
$$

On the other hand, the function $z:=w_{x}$ satisfies $z_{t}=z_{x x}+a(t, x) z_{x}$ in $(0, T) \times \mathbb{R}$, where $a(t, x)=p\left|w_{x}\right|^{p-2} w_{x}$. By a further application of the maximum principle, we deduce that

$$
\left|w_{x}\right| \leq\left|w_{0, x}\right|_{\infty} \quad \text { on }(0, T) \times \mathbb{R}
$$

This, along with (2.7), implies in particular that $T=\infty$.

Let now $A=\left|w_{0, x}\right|_{\infty}^{p-2}$. Due to (2.9), we have $w_{t} \leq w_{x x}+A\left|w_{x}\right|^{2}$ and the function $y:=e^{A w}-1 \geq 0$ thus satisfies $y_{t} \leq y_{x x}$ in $(0, \infty) \times \mathbb{R}$. It follows that $y(t) \leq G_{t} * y(0)$, where $G_{t}(x)=(4 \pi t)^{-1 / 2} \exp \left[-x^{2} / 4 t\right]$. Therefore, for all $t>0$, we get

$$
A|w(t)|_{\infty} \leq\left|e^{A w(t)}-1\right|_{\infty} \leq(4 \pi t)^{-1 / 2}\left|e^{A w_{0}}-1\right|_{L^{1}(\mathbb{R})},
$$

hence $|w(t)|_{\infty} \leq C t^{-1 / 2}$. In view of (2.8), this yields the Lemma.

Proof of Lemma 2.5. Assume that the Lemma is false. Then, by Lemma 2.3, there exists a sequence $t_{n} \rightarrow \infty$ such that $u_{x}\left(t_{n}, 1\right) \rightarrow-\infty$.

Fix $\varepsilon>0$. By (2.6) in Lemma 2.4, for $n \geq n_{0}(\varepsilon)$ large enough, we have

$$
\left[\left(-u_{x}\right)^{+}\left(t_{n}, 1-x\right)+C_{3}\right]^{1-p} \leq p \varepsilon, \quad 0 \leq x \leq \varepsilon
$$

hence,

$$
\left(-u_{x}\right)^{+}\left(t_{n}, 1-x\right) \geq(p \varepsilon)^{-1 /(p-1)}-C_{3}, \quad 0 \leq x \leq \varepsilon .
$$

By choosing $\varepsilon=\varepsilon\left(p, C_{3}\right)>0$ small, we deduce that $u_{x}\left(t_{n}, 1-x\right) \leq-1$ on $[0, \varepsilon]$, hence

$$
u\left(t_{n}, 1-x\right) \geq M+x, \quad 0 \leq x \leq \varepsilon
$$

for all $n \geq n_{0}(\varepsilon)$. But this contradicts Lemma 2.6.

Lemma 2.5 is already enough to conclude in the case $M=0$.

Proof of Theorem 1 For $M=0$. Let $u$ be a global solution of (1.1). Since $M=0$, we observe that $v(t, x):=u(t, 1-x)$ solves $(1.1)$ with $u_{0}$ replaced by $u_{0}(1-x)$. Therefore, Lemma 2.5 implies that both $u_{x}$ and $v_{x}$ are bounded below on $[0, \infty) \times[0,1]$, which means that $u_{x}$ is bounded. (For the convergence of $u$ to $V_{0}=0$, see the end of the proof of the case $M>0$ below.) 


\section{3. - Lyapunov functional and proof of Theorem 1 for $M>0$}

As a main step, we now carry out the argument of Zelenyak to construct a Lyapunov functional. The key point here is that the Lyapunov functional enjoys nice properties on any global trajectory of (1.1), even if it were unbounded in $C^{1}$.

Proposition 3.1. Fix any $K>0$ and let $D_{K}=[-K, K] \times \mathbb{R}$. There exist functions $\phi \in C^{1}\left(D_{K} ; \mathbb{R}\right)$, and $\psi \in C\left(D_{K} ;(0, \infty)\right)$ with the following property. For any solution $u$ of (1.1) with $|u| \leq K$, defining

$$
\mathcal{L}(u(t)):=\int_{0}^{1} \phi\left(u(t, x), u_{x}(t, x)\right) d x
$$

it holds

$$
\frac{d}{d t} \mathcal{L}(u(t))=-\int_{0}^{1} \psi\left(u(t, x), u_{x}(t, x)\right) u_{t}^{2}(t, x) d x, \quad 0<t<T^{*}
$$

Furthermore, we have

$$
\phi \geq 0
$$

As a consequence of Proposition 3.1 and of the estimates of Section 2, we shall obtain the following convergence result. Of course, the main point here is that we do not assume $u$ to be bounded, but only global (since otherwise the statement is nothing more but the result of [30], and the convergence even holds in $C^{1}$ ).

Proposition 3.2. Let $u$ be a global solution of (1.1). Then, as $t \rightarrow \infty$, $u(t)$ converges in $C([0,1])$ to a stationary solution of $(1.1)$, i.e. a function $W \in$ $C([0,1]) \cap C^{2}(0,1)$ of

$$
\left\{\begin{array}{l}
W_{x x}+\left|W_{x}\right|^{p}=0, \\
W(0)=0, \quad W(1)=M
\end{array}\right.
$$

Moreover, the convergence also holds in $C^{1}([\varepsilon, 1])$ for all $\varepsilon>0$.

Proof of Proposition 3.1. For a given function $\varphi(u, v)$, let us denote

$$
H=\varphi_{u}+|v|^{p} \varphi_{v v}-v \varphi_{v u}
$$

Here we assume that $\varphi, \varphi_{u}, \varphi_{v}, \varphi_{v u}$ are continuous and $C^{1}$ in $v$ in $D_{K}$, and that $\varphi_{v v}$ is continuous in $D_{K}$ and, except perhaps at $v=0, C^{1}$ in $v$. We observe that $H$ is continuous and differentiable in $v$ in $D_{K}$ and satisfies

$$
H_{v}=|v|^{p} \varphi_{v v v}+p|v|^{p-2} v \varphi_{v v}-v \varphi_{v u v} \quad\left(H_{v}=0 \text { for } v=0\right) .
$$


Now suppose that $\psi:=\varphi_{v v}$ satisfies

$$
\psi_{u}-|v|^{p-2} v \psi_{v}-p|v|^{p-2} \psi=0, \quad|u| \leq K, \quad v \neq 0 .
$$

It follows that $H_{v}=0$, hence

$$
H=H(u)=\varphi_{u}(u, 0)
$$

Let then

$$
\phi(u, v)=\varphi(u, v)-\int_{0}^{u} H(s) d s=\varphi(u, v)-\varphi(u, 0)+\varphi(0,0) .
$$

We compute, using integration by parts and $u_{t}(t, 0)=u_{t}(t, 1)=0$,

$$
\begin{aligned}
\frac{d}{d t} \mathcal{L}(u(t)) & =\int_{0}^{1}\left\{\left(\varphi_{u}\left(u, u_{x}\right)-H(u)\right) u_{t}+\varphi_{v}\left(u, u_{x}\right) u_{x t}\right\}(t, x) d x \\
& =\int_{0}^{1}\left\{\left(\varphi_{u}\left(u, u_{x}\right)-H(u)-\varphi_{v u}\left(u, u_{x}\right) u_{x}-\varphi_{v v}\left(u, u_{x}\right) u_{x x}\right\} u_{t}(t, x) d x .\right.
\end{aligned}
$$

Using the definition of $H$ and $u_{x x}=u_{t}-\left|u_{x}\right|^{p}$, we deduce that

$$
\frac{d}{d t} \mathcal{L}(u(t))=-\int_{0}^{1} \psi\left(u(t, x), u_{x}(t, x)\right) u_{t}^{2}(t, x) d x
$$

We have thus obtained (3.1), provided (3.4) is true.

Now, the equation (3.4) can be solved by the method of characteristics. For each $K>0$, one finds that the function $\psi$ defined by

$$
\psi(u, v)=\left[1+(p-2)|v|^{p-2}(K+1-u)\right]^{-p /(p-2)}>0
$$

is a solution of $(3.4)$ on $[-K, K] \times(\mathbb{R}-\{0\})$.

Define $\varphi$ by

$$
\varphi(u, v)=\int_{0}^{v} \int_{0}^{z} \psi(u, s) d s d z \geq 0 .
$$

It is easy to check that $\varphi$ enjoys the regularity properties assumed at the beginning of the proof and $\phi=\varphi$, hence (3.2). 
Proof of Proposition 3.2. Fix any sequence $t_{n} \rightarrow \infty$ and let $u_{n}=$ $u\left(t_{n}+.,.\right)$. Denote $Q:=[0, \infty) \times(0,1]$ and $Q_{\varepsilon}:=[0, \infty) \times(\varepsilon, 1]$, for all $\varepsilon>0$.

From (1.2) and Lemma 2.1, we know that

$$
|u|+\left|u_{t}\right| \leq C \quad \text { in }[1, \infty) \times[0,1] .
$$

Also, using (2.1), $p>2$ and Lemma 2.5, we obtain

$$
\left|\partial_{x} u_{n}\right|_{L^{\infty}\left(1, \infty ; L^{1}(0,1)\right)} \leq C .
$$

It follows from (3.5) and (3.6) that

(3.7) the sequence $\left(u_{n}\right)$ is relatively compact in $C([0, T] \times[0,1])$ for each $T>0$.

On the other hand, using (2.1), (2.2) and (3.5), we have

$$
\left|u_{x}\right| \leq C(\varepsilon) \quad \text { and hence } \quad\left|u_{x x}\right| \leq C(\varepsilon) \quad \text { in }[1, \infty) \times(\varepsilon, 1] .
$$

Since $w:=u_{x}$ satisfies $w_{t}-w_{x x}=p\left|u_{x}\right|^{p-2} u_{x} u_{x x}$, parabolic regularity estimates then imply that for each $q>1$,

$$
\left|w_{t}\left(t_{n}+., .\right)\right|_{L^{q}((0, T) \times(\varepsilon, 1))} \leq C(\varepsilon, T, q), \quad T>0 .
$$

It follows from (3.8) and (3.9) that the sequence $\left(\partial_{x} u_{n}\right)$ is relatively compact in $C([0, T] \times[\varepsilon, 1])$ for each $\varepsilon, T>0$. This, together with (3.7), implies that some subsequence $\left(u_{n_{k}}\right)$ converges to a function $W \in C(\bar{Q})$, with $W_{x} \in C(Q)$, which satisfies

$$
\begin{cases}W_{t}-W_{x x}=\left|W_{x}\right|^{p} & \text { in } Q \\ W(t, 0)=0, \quad W(t, 1)=M, & t \geq 0\end{cases}
$$

(The convergence of $u_{n_{k}}$ is uniform in each set $[0, T] \times[0,1]$ and the convergence of $\partial_{x} u_{n_{k}}$ is uniform in each set $[0, T] \times[\varepsilon, 1]$.)

Now, by (1.2) we may find $K>0$ such that

$$
|u| \leq K \quad \text { on }[0, \infty) \times[0,1] .
$$

Since $\psi$, given by Proposition 3.1, is positive continuous, we have (3.10) $\quad \eta(K, R):=\inf \{\psi(u, v) ;|u| \leq K,|v| \leq R\}>0, \quad$ for all $R>0$. 
Fix any $\varepsilon \in(0,1)$. By (3.1), (3.8), (3.10) and (3.2), we get, for all $T>1$,

$$
\begin{aligned}
\eta(K, C(\varepsilon)) \int_{1}^{T} \int_{\varepsilon}^{1} u_{t}^{2}(t, x) d x d t & \leq \int_{1}^{T} \int_{0}^{1} \psi\left(u, u_{x}\right) u_{t}^{2}(t, x) d x d t \\
& =\mathcal{L}(u(1))-\mathcal{L}(u(T)) \leq \mathcal{L}(u(1)) .
\end{aligned}
$$

This implies that $\int_{1}^{\infty} \int_{\varepsilon}^{1} u_{t}^{2}(t, x) d x d t<\infty$, hence

$$
\int_{0}^{\infty} \int_{\varepsilon}^{1}\left(\partial_{t} u_{n_{k}}\right)^{2}(t, x) d x d t=\int_{t_{n_{k}}}^{\infty} \int_{\varepsilon}^{1} u_{t}^{2}(t, x) d x d t \rightarrow 0, \quad k \rightarrow \infty .
$$

Since $\partial_{t} u_{n_{k}} \rightarrow W_{t}$ in $\mathcal{D}^{\prime}((0, \infty) \times(0,1))$ and since $\varepsilon \in(0,1)$ is arbitrary, it follows that $W_{t} \equiv 0$. Therefore, $W=W(x) \in C([0,1]) \cap C^{2}(0,1)$ satisfies (3.3).

But we know (cf. the beginning of Section 2) that the solution of (3.3) is unique whenever it exists. Since the sequence $t_{n} \rightarrow \infty$ was arbitrary, this readily implies that the whole solution $u(t)$ actually converges to $W$. The Proposition is proved.

Proof of TheOREM 1 FOR $0<M<M_{c}$. Assume that $u$ is a global solution of (1.1) which is unbounded in $C^{1}$. By Proposition 3.2, as $t \rightarrow \infty, u(t)$ converges to $W=V_{M}$, with convergence in $C([0,1])$ and in $C^{1}([\varepsilon, 1])$ for all $\varepsilon>0$.

Since $u$ is unbounded, by Lemmas 2.3 and 2.5 , there exists a sequence $t_{n} \rightarrow \infty$ such that

$$
u_{x}\left(t_{n}, 0\right) \rightarrow \infty
$$

Using Lemma 2.5, (2.5) and (3.11), we deduce that $W_{x}(x) \geq-C$ and $\left[W_{x}^{+}(x)+\right.$ $\left.C_{3}\right]^{1-p} \leq(p-1) x$ in $(0,1]$, This easily implies that

$$
W_{x}(x) \geq((p-1) x)^{-\frac{1}{p-1}}-C^{\prime} \quad \text { in }(0,1] .
$$

But this is a contradiction, since $W=V_{M} \in C^{1}([0,1])$. We have thus proved that all global solutions are bounded in $C^{1}$.

Finally, once boundedness is known, the convergence of global solutions to $V_{M}$ in $C^{1}$ (also for $M=0$ ) is a standard consequence of the existence of a Lyapunov functional, the uniqueness of the steady-state, and compactness properties of the semi-flow associated with (1.1). The proof of Theorem 1 is complete.

Proof of Proposition 2. This is an immediate consequence of Proposition 3.2 and the fact that (3.3) admits no solution for $M>M_{c}$.

Proof of Proposition 3. Let

$$
D=\left\{u_{0} \in X ; u\left(u_{0} ; t, .\right) \text { converges to } V_{M} \text { in } C^{1} \text { as } t \rightarrow \infty\right\}
$$


and fix $\bar{M} \in\left(M, M_{c}\right)$. We claim that:

$$
\text { for all } u_{0} \in X, u_{0} \leq \min \left(M, V_{\bar{M}}\right) \text { implies } u_{0} \in D \text {. }
$$

Indeed, by the comparison principle, as long as $u:=u\left(u_{0} ; t,.\right)$ exists, we have $u \leq V_{\bar{M}}$, hence $u_{x}(t, 0) \leq V_{\bar{M}, x}(0)$, and $u \leq M$, hence $u_{x}(t, 1) \geq 0$. By Lemma 2.3, we deduce that $u$ is global and bounded in $C^{1}$. It then follows from [30] that $u$ converges in $C^{1}$ to the unique steady state $V_{M}$ as $t \rightarrow \infty$, which proves the claim.

Let us first consider the case $M \in\left(0, M_{c}\right)$. By [2, Theorem 1.2], there exists $\bar{u}_{0} \in X$ with $\bar{u}_{0, x} \geq 0$, such that $T^{*}\left(\bar{u}_{0}\right)<\infty$. For each $\lambda \in[0,1]$, denote $u_{0, \lambda}:=V_{M}+\lambda\left(\bar{u}_{0}-V_{M}\right) \in X$ and $u_{\lambda}:=u\left(u_{0, \lambda} ; t,.\right)$. For $\lambda>0$ small, we have $u_{0, \lambda} \leq \min \left(M, V_{\bar{M}}\right)$, hence $u_{0, \lambda} \in D$. Therefore $\lambda^{*}:=\inf \left\{\lambda \in[0,1] ; u_{0, \lambda} \notin\right.$ $D\} \in(0,1]$. By (3.12) and a standard continuous dependence argument, we have $u_{0, \lambda^{*}} \notin D$. This implies that $u_{\lambda^{*}}$ cannot be global and bounded in $C^{1}$ (since otherwise it would converge to $V_{M}$ due to [30]). In view of Theorem 1 , the only remaining possibility is that $T^{*}\left(u_{0, \lambda^{*}}\right)<\infty$. Considering $u_{0, \lambda_{n}}$ for a sequence $\lambda_{n} \uparrow \lambda^{*}$, we obtain the conclusions (i) and (ii) of Proposition 3. We also get (iii), since otherwise $u_{\lambda^{*}}$ would be global by continuous dependence.

In the case $M=0$, we fix any $\phi \in X$ with $\phi \geq 0, \phi \not \equiv 0$, and we set $u_{0, \lambda}=\lambda \phi$. We have $T^{*}\left(u_{0, \lambda}\right)<\infty$ for $\lambda>0$ large by [28, Theorem 2.1], whereas $u_{0, \lambda} \in D$ for $\lambda>0$ small (indeed, by comparing with $V_{M}$ and $V_{M}(1-x)$ for some $M \in\left(0, M_{c}\right)$, one sees that $u$ remains bounded in $\left.C^{1}\right)$. The rest of the proof is then similar.

\section{REFERENCES}

[1] N. AlaA, Solutions faibles d'équations paraboliques quasi-linéaires avec données initiales mesures, Ann. Math. Blaise-Pascal 3 (1996) 1-15.

[2] N. Alikakos - P. Bates - C. Grant, Blow up for a diffusion-advection equation, Proc. Roy. Soc. Edinburgh A 113 (1989), 181-190.

[3] S. Angenent - M. Fila, Interior gradient blow-up in a semilinear parabolic equation, Differential Integral Equations 9 (1996), 865-877.

[4] G. BARLES - F. DA Lio, On the generalized Dirichlet for viscous Hamilton-Jacobi equations, J. Math. Pures et Appl., to appear.

[5] M. Benachour - S. Dabuleanu, The mixed Cauchy-Dirichlet for a viscous HamiltonJacobi equation, Adv. Differential Equations, to appear.

[6] M. Ben-Artzi - Ph. Souplet - F. B. Weissler, The local theory for viscous HamiltonJacobi equations in Lebesgue spaces, J. Math. Pures et Appl. 81 (2002) 343-378. 
[7] T. Cazenave - P.-L. Lions, Solutions globales d'equations de la chaleur semilinéaires, Commun. Partial Differential Equations 9 (1984), 955-978.

[8] C.-N. Chen, Infinite time blow-up of solutions to a nonlinear parabolic problem, J. Differential Equations 139 (1997), 409-427.

[9] G. Conner - C. Grant, Asymptotics of blowup for a convection-diffusion equation with conservation, Differential Integral Equations 9 (1996), 719-728.

[10] S. Dabuleanu, "Problèmes aux limites pour les équations de Hamilton-Jacobi avec viscosité et données initiales peu regulières", PhD thesis, Université Nancy 1, 2003.

[11] K. Deng, Stabilization of solutions of a nonlinear parabolic equation with a gradient term, Math. Z., 216 (1994), 147-155.

[12] M. Fila - B. Kawohl, Is quenching in infinite time possible ?, Quart. Appl. Math. 8 (1990), 531-534.

[13] M. Fila - G. Lieberman, Derivative blow-up and beyond for quasilinear parabolic equations, Differential Integral Equations 7 (1994), 811-821.

[14] M. Fila - P. SACKs, The transition from decay to blow-up in some reaction-diffusionconvection equations, World Congress of Nonlinear Analysts '92, Vol. I-IV (Tampa, FL, 1992), 455-463, de Gruyter, Berlin, 1996.

[15] M. Fila - PH. Souplet - F. B. WeISSLER, Linear and nonlinear heat equations in $L_{\delta}^{q}$ spaces and universal bounds for global solutions, Math. Ann. 320 (2001), 87-113.

[16] V. Galaktionov - J.-L. Vázquez, Continuation of blow-up solutions of nonlinear heat equations in several space dimensions, Comm. Pure Appl. Math. 50 (1997), 1-67.

[17] Y. Giga, A bound for global solutions of semi-linear heat equations, Comm. Math. Phys. 103 (1986), 415-421.

[18] M. Kardar - G. Parisi - Y. C. Zhang, Dynamic scaling of growing interfaces, Phys. Rev. Lett. 56 (1986), 889-892.

[19] O. Ladyzenskaya - V. A. Solonnikov - N. N. Uralceva, "Linear and Quasilinear Equations of Parabolic Type”, Amer. Math. Soc. Translations, Providence, RI, 1967.

[20] G. Lieberman, The first initial-boundary value problem for quasilinear second order parabolic equations, Ann. Scuola Norm. Sup. Pisa Cl. Sci. 13 (1986), 347-387.

[21] P. L. Lions, "Generalized solutions of Hamilton-Jacobi Equations", Pitman Research Notes in Math. 62, 1982.

[22] W.-M. Ni - P. E. SACKS - J. TAVANTZis, On the asymptotic behavior of solutions of certain quasi-linear equations of parabolic type, J. Differential Equations 54 (1984), 97-120.

[23] P. QuiTTNER, A priori bounds for global solutions of a semilinear parabolic problem, Acta Math. Univ. Comenianae 68 (1999), 195-203.

[24] P. QUITTNER, Universal bound for global positive solutions of a superlinear parabolic problem, Math. Ann. 320 (2001), 299-305.

[25] P. QuitTNER, Continuity of the blow-up time and a priori bounds for solutions in superlinear parabolic problems, Houston J. Math. 29 (2003), 757-799.

[26] P. QuittNer - Ph. SOuplet - M. Winkler, Initial blow-up rates and universal bounds for nonlinear heat equations, J. Differential Equations, to appear.

[27] $\mathrm{PH}$. Souplet, Recent results and open problems on parabolic equations with gradient nonlinearities, Electronic J. Differential Equations 20 (2001), 1-19.

[28] Рн. Souplet, Gradient blow-up for multidimensional nonlinear parabolic equations with general boundary conditions, Differential Integral Equations 15 (2002), 237-256. 
[29] Рh. Souplet - F. B. WeissLer, Poincaré's inequality and global solutions of a nonlinear parabolic equation, Ann. Inst. H. Poincaré, Anal. Non linéaire 16 (1999), 337-373.

[30] T. I. ZelenyaK, Stabilisation of solutions of boundary value problems for a second-order equation with one space variable, Differential Equations 4 (1968), 17-22.

Departamento de Matemática Aplicada

Universidad Complutense

28040 Madrid, Spain

jose.arrieta@mat.ucm.es

arober@mat.ucm.es

Département de Mathématiques

INSSET

Université de Picardie

02109 St-Quentin, France

and

Laboratoire de Mathématiques Appliquées

UMR CNRS 7641

Université de Versailles

45 avenue des Etats-Unis

78035 Versailles, France

souplet@math.uvsq.fr 